\title{
DEPRESSÃO INFANTIL E DESENVOLVIMENTO PSICOCOGNITIVO: DESCRIÇÃO DAS RELAÇÕES DE CAUSALIDADE
}

\section{CHILDHOOD DEPRESSION AND PSYCHOCOGNITIVE DEVELOPMENT: DESCRIPTION OF CAUSALITY RELATIONSHIPS}

\author{
Modesto Leite Rolim Neto ${ }^{1,2}$, Thalita do Nascimento Silva ${ }^{1}$, \\ José Kleber Mota Assunção Filho ${ }^{1}$, Rebeca de Sousa Carvalho', \\ Saulo Araújo Teixeira', Nádia Nara Rolim Lima ${ }^{1}$, Daniela Pedroso", \\ Jesus de Souza Cartaxoㄹ, Jesualdo Alves Duarte Júnior ${ }^{1}$, \\ Marcelo Marcos Piva Demarzo ${ }^{3}$, Alberto Olavo Advíncula Reis ${ }^{2}$
}

\begin{abstract}
Resumo:
Alterações de humor e distúrbios de saúde mental têm reconhecida influência no comportamento social, familiar e escolar de crianças e adolescentes. A incidência desses transtornos tem apresentado a tendência de aumento em todo o mundo e seu diagnóstico precoce aventa discussões a respeito da interferência dessas patologias no processo natural de desenvolvimento psicocognitivo de seus portadores. Este estudo pretende descrever como a depressão infantil interfere no desenvolvimento cognitivo, a partir de alterações na esfera psicocognitiva. Para tal, foram consultadas as bases de dados da SciELO e da Biblioteca Virtual de Saúde (BVS) no período de 2006 a 2011, utilizando os termos "depressão", "infantil" e "desenvolvimento". Após seleção dos resultados, oito artigos foram usados para a elaboração deste trabalho. Os resultados evidenciaram que a depressão infantil repercute negativamente no desenvolvimento cognitivo infantil. Transtornos psíquicos fazem a criança reconhecer-se como incapaz e expressar sentimentos de vergonha, indefinição, baixos níveis de autoestima e distanciamento das demandas da aprendizagem. O profissional de educação deve estar apto a reconhecer essas alterações manifestadas no ambiente escolar. A análise dos estudos mostrou que um ambiente externo saudável possibilita adequada maturação do sistema cognitivo e protege a criança da depressão infantil. Enquanto a maioria dos estudos sugere relação causal entre depressão e dificuldade cognitiva, outros estudos mostram a relação inversa, em que a baixa cognição gera sintomas de depressão. A dificuldade de aprendizagem e a depressão infantil podem coexistir sem que haja, necessariamente, uma relação de causalidade entre si. Dessa forma, faz-se necessário o diagnóstico precoce a fim de que as modificações do desenvolvimento cognitivo possam ser reparadas, sem maiores injúrias para as crianças acometidas.
\end{abstract}

Palavras-chave: depressão; desenvolvimento infantil; depressão infantil. Abstract

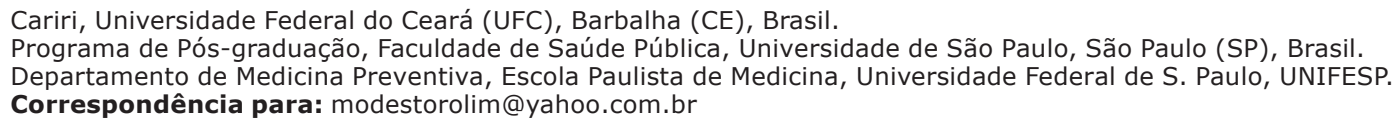

Fontes de Financiamento

Fundação Cearense de Apoio ao Desenvolvimento Científico e Tecnológico (FUNCAP). Universidade Federal do Ceará (UFC).

Como citar este artigo: Rolim Neto ML, et al. Childhood depression and psychocognitive development: description of causality relationships. Journal of Human Growth and Development 2011; 21(3): 894-898.

Artigo submetido em 06.08.10, aceito em 30.09.11. 


\begin{abstract}
Mental health disorders provoke important changes on children behavior. The incidence of these pathologies has increased worldwide and they are diagnosed earlier, what start a discussion about their influence on psychosocial development of carrier children. We wanted to describe how depression affects child cognitive development, with changes in cognitive psycho-sphere. Two databases (SciELO and BVS) were surveyed between 2006 and 2011, using the keywords "depression", "children" and "development". After analysis, eight articles were selected and used to construct this work. Results disclose that childhood depression is a negative influence to children cognitive development. In case of illness, child recognizes itself as unable to achieve demands which are betting on it, with feelings of shame, doubt, lack of self-confidence and learning problems, being unable to "make their best". Education professional must be able to recognize these changes manifest in the school environment. Studies showed that a healthy external environment allows for a proper maturation of the cognitive system protects children and childhood depression. While most studies suggest a causal relationship between depression and cognitive impairment, other studies show the inverse relationship in the low cognition generates symptoms of depression. However, it should also be noted that difficulty of learning and childhood depression can coexist without there necessarily a causal relationship between them. It is necessary to diagnose earlier these disorders, making possible to repair injuries in children development.
\end{abstract}

Key words: depression; child development; childhood depression.

\section{INTRODUÇÃO}

A pesquisa na área da saúde mental tem, desde tempos recentes, alcançado destaque, impulsionada principalmente pela literatura, que é enfática em sublinhar aumento na prevalência dos transtornos psiquiátricos na infância e na adolescência (oscilação entre 1 a 51\% na prevalência destes) ${ }^{1}$.

Dentre essas patologias psiquiátricas que acometem crianças, merece atenção especial a depressão infantil, devido às potenciais implicações danosas que essa entidade gera no desenvolvimento humano. Esse transtorno de humor tem sido caracterizado por sintomas como irritabilidade, reclamações somáticas, reclusão do convívio social e humor diminuído, o que levou a reconhecê-lo como uma doença distinta daquela que ocorre no adulto. Esses sintomas, em curto prazo, podem atuar como fontes de sofrimento psíquico para essas crianças e, em longo prazo, podem interferir nos aspectos cognitivo, social e emocional do desenvolvimento infantil2.

A depressão infantil é uma patologia que tanto pode interferir como se manifestar nos aspectos físicos, comportamentais, cognitivos e sociais, permitindo compreendê-la como um fenômeno biopsicossocial. Essas alterações sobre as esferas psicoafetiva, psicossocial, orgânica, humoral e psicocognitiva, ocorrem em uma frequência de $31,42 \%$; $31,42 \% ; 19,70 \% ; 13,97 \%$ e $3,49 \%$, respectivamente ${ }^{3}$.

A esfera psicocognitiva, apesar de apresentar uma menor percentagem de frequência em relação às demais, merece uma abordagem mais contundente pelo meio científico, já que alterações significantes nesse aspecto tendem a repercutir negativamente no desenvolvimento da criança. Tais alterações podem ser observadas principalmente no ambiente escolar, na forma de dificuldades para se concentrar e para pensar.

As alterações psicocognitivas possuem tendência de provocar evitação do trabalho escolar, levando a criança ao baixo rendimento acadêmico. Assim, objetiva-se descrever as formas de relações causais existentes entre depressão na infância e o desenvolvimento dessas crianças. 


\section{MÉTODO}

Trata-se de uma revisão narrativa, em que foram consultadas as bases de dados SciELO e BVS no período de 2006 a 2011. Na busca, foram utilizados os descritores "depressão", "infantil" e "desenvolvimento" em conjunto, para encontrar os trabalhos que contivessem o termo em qualquer campo. A pesquisa retornou 14 artigos que versavam sobre essa temática. Além desses, foram adicionados ao estudo outros artigos e livros de referência, com abordagem pertinente e não pertencentes às bases de dados SciELO e BVS.

A seleção dos textos foi realizada manualmente, sendo excluídos aqueles não relacionados à depressão e ao desenvolvimento infantil ou que apresentavam enfoque diverso dos objetivos deste trabalho. Estudos de natureza qualitativa e quantitativa, em dois idiomas (português e inglês) foram considerados, num total de oito artigos que alicerçaram os resultados e a discussão.

\section{RESULTADOS}

A literatura contemplada descreve de forma incisiva que a depressão infantil repercute negativamente no desenvolvimento cognitivo. Contudo, nem sempre essa alteração torna-se perceptível, já que a criança, na maioria das vezes, não consegue expressar o que está sentindo na forma verbal. A fase do desenvolvimento psicocognitivo que essa criança vivencia é determinante em moldar as suas formas de expressão ${ }^{4}$.

As dificuldades escolares podem ser consideradas, muitas vezes, como o primeiro sinal de que a criança está iniciando um quadro de depressão ${ }^{5}$. Nesse processo de adoecimento, a criança reconhece-se como incapaz de atender às exigências impostas a ela, evidenciando sentimentos de vergonha, indefinição e distanciamento das demandas da aprendizagem, com consequente prejuízo da autoestima. Essas manifestações tendem a determinar baixo rendimento escolar, tanto na fase pueril, como no futuro educacional dessa criança6.

Descrevendo as funções cognitivas que são afetadas no processo depressivo, alguns autores referem que, além de dificuldades na concentração e na atenção, a memória e o raciocínio também estão alterados, com consequente influência negativa no desempenho escolar da criança 7,8. Há impossibilidade de "dar 0 melhor de si".

No entanto, deve-se estar atento que de forma alguma tais sintomas podem ser considerados isoladamente, mas, sim, deve haver uma análise simultânea no que diz respeito à sua intensidade e a seu tempo de duração ${ }^{9}$.

Um fato importante é que crianças, nessa faixa etária, tendem a dedicar, ainda que não espontaneamente, tempo maior ao ambiente escolar comparado à convivência em família ${ }^{10}$. Assim, o profissional pedagogo, que possui ampla bagagem de conhecimentos sobre as fases do processo de desenvolvimento infantil e sobre as principais manifestações sintomatológicas da doença em questão, deverá estar apto a reconhecer de forma precoce essa enfermidade, permitindo que haja intervenção terapêutica resolutiva em curto prazo, a fim de minorar as repercussões da depressão infantil no desenvolvimento cognitivo em longo prazo ${ }^{4,11}$.

\section{DISCUSSÃo}

A criança, ao longo do seu desenvolvimento cognitivo, sofre profundas modificações em determinadas áreas cerebrais, de modo que a presença de eventos estressantes vivenciados precocemente são fatores de grande influência para um desenvolvimento cerebral inadequado ${ }^{5}$. Assim, fica evidente a necessidade da existência de um ambiente externo saudável que tanto possibilite uma adequada maturação do sistema cognitivo, como sirva de proteção ao desenvolvimento da doença depressiva.

Em cada fase do desenvolvimento infantil a criança pode apresentar uma sintomatologia específica que, muitas 
vezes, pode ser bem distinta de outra fase. Assim, faz-se necessário que os pedagogos saibam as características do desenvolvimento cognitivo normal de cada fase, a fim de que o patológico torne-se perceptível. Dessa forma, será possível reconhecer, no período escolar, quando a redução do rendimento educacional infantil tem relação causal direta com uma manifestação do quadro depressivo ${ }^{4}$. Essa identificação precoce permitirá prevenir maiores repercussões negativas sobre o desenvolvimento cognitivo de crianças depressivas.

Quando se fala da associação entre depressão e problemas de aprendizagem, coloca-se mais ênfase na relação causal em que a depressão infantil leva a dificuldades escolares. Contudo, é preciso levar em consideração que essas crianças também podem ser portadoras de outra comorbidade psiquiátrica que afete seu desenvolvimento cognitivo ${ }^{6}$, não sendo possível nesses casos estabelecer uma relação causal direta entre depressão e dificuldade escolar.

Uma discussão que pode ser observada na literatura é se as crianças deprimidas apresentam ou não um déficit intelectual. $\mathrm{O}$ que alguns estudos sugerem é que a inteligência não se relaciona diretamente com a depressão, isto é, a criança deprimida não apresenta, necessariamente, um nível intelectual abaixo da média. Dito de outra forma, a criança não consegue utilizar todo o seu potencial cognitivo por ter as manifestações depressivas como um obstáculo ao seu pleno funcionamento ${ }^{11}$.

\section{REFERÊNCIAS}

1. Assis SG, Avanci JQ, Pesce RP, Ximenes LF. The situation of Brazilian children and adolescents with regard to mental health and violence. Ciênc. saúde coletiva. 2009;14(2):349-361.

2. Oliveira JSC, Ribeiro KCL, Araújo LF, Coutinho MPL. Social representations of depression by children with depressive symptomatology. Adv. health psych. 2009;14(2):160-170.
É nesse contexto que se reconhece o importante papel que deve ser exercido pelos pedagogos. Esses profissionais estabelecem um contato mais duradouro e próximo com as crianças ${ }^{12}$. Entende-se assim que uma melhor capacitação desse profissional permitirá sua ação como importante instrumento no screening da depressão infantil, por meio da percepção das alterações nas diversas fases do desenvolvimento cognitivo infantil.

Por fim, a depressão infantil interfere de forma substancial no desenvolvimento cognitivo da criança. Familiares, pedagogos, médicos, terapeutas e demais profissionais que estejam envolvidos com a criança devem atentar-se para as dificuldades educacionais, como fontes de suspeição precoce da depressão infantil, visando a uma intervenção nesse processo de adoecimento, reduzindo principalmente os déficits da função intelectual.

As dificuldades cognitivas causadas pela depressão infantil podem repercutir negativamente, tanto em termos financeiros quanto sociais, pois a criança com tais limitações tende a se tornar um adulto com desenvolvimento intelectual comprometido, dificultando sua aceitação no mercado de trabalho e a construção de vínculos sociais sólidos.

\section{AGRADECIMENTOS}

À Universidade Federal do Ceará (UFC) e à Fundação Cearense de Apoio ao Desenvolvimento Científico e Tecnológico (FUNCAP), pelo suporte financeiro.

3. Couto MCV, Duarte CS, Delgado PGG. Child mental health and public health in Brazil: current situation and challenges. Rev. bras. psiquiatr. 2008;30(4):384-389.

4. Fernandes AM, Milani RG. A depressão infantil, o rendimento escolar e a autoeficácia: uma revisão da literatura. Rev. cesumar. 2010; 30(4): 384-389.

5. Santos BS. Uma cartografia simbólica das representações sociais. Rev Crít Ciênc Soc 1988;24:139-172. 
6. Jamison KR. Uma mente inquieta: memórias de loucura e instabilidade de humor. São Paulo: Martins Fontes; 1996. pp.46-9.

7. Oliveira PA, Scivoletto S, Cunha PJ. Neuropsychological and neuroimaging studies associated with emotional stress during childhood and adolescence. Rev. psiquiatr. clín. 2010;37(6):271-279.

8. Solomon A. Demônio do meio-dia: uma anatomia da depressão. Rio de Janeiro: Objetiva; 2002. pp.38-9.

9. Allen-Meares $P$, Colarossi L, Oyserman D, DeRoos Y. Assessing depression in childhood and adolescence: a Guide for social work practice child and adolescent. Social Work Journal. 2003;20(1):5-20.

10. Cruvinel M, Boruchovitch E. Depressive symptoms, learning strategies and academic achievement among elementary school students. Psicol. estudo. 2004;9(3):369-378.

11. Cowan CP, Cowan PA, Pruett MK, Pruett $\mathrm{K}$. An approach to preventing coparenting conflict and divorce in lowincome families: strengthening couple relationships and fostering father's involvement. Family process. 2008;46(1):109-121.

12. Avanci J, Assis S, Oliveira R, Pires T. When living with violence brings a child close to depressive behavior. Ciênc. saúde coletiva. 2009; 14(2): 383-394. 